\title{
Metanalysis: building evidence-based speech therapy
}

\author{
Vanessa Souza Gigoski de Miranda ${ }^{1}$ \\ https://orcid.org/0000-0002-3332-9975 \\ Miriam Allein Zago Marcolino ${ }^{1}$ \\ https://orcid.org/0000-0001-5333-3173 \\ Rafaela Soares Rech ${ }^{1}$ \\ https://orcid.org/0000-0002-3207-0180
}

Universidade Federal de Ciências da Saúde de Porto Alegre, Porto Alegre, Rio Grande do Sul, Brasil.

Conflict of interests: Nonexistent

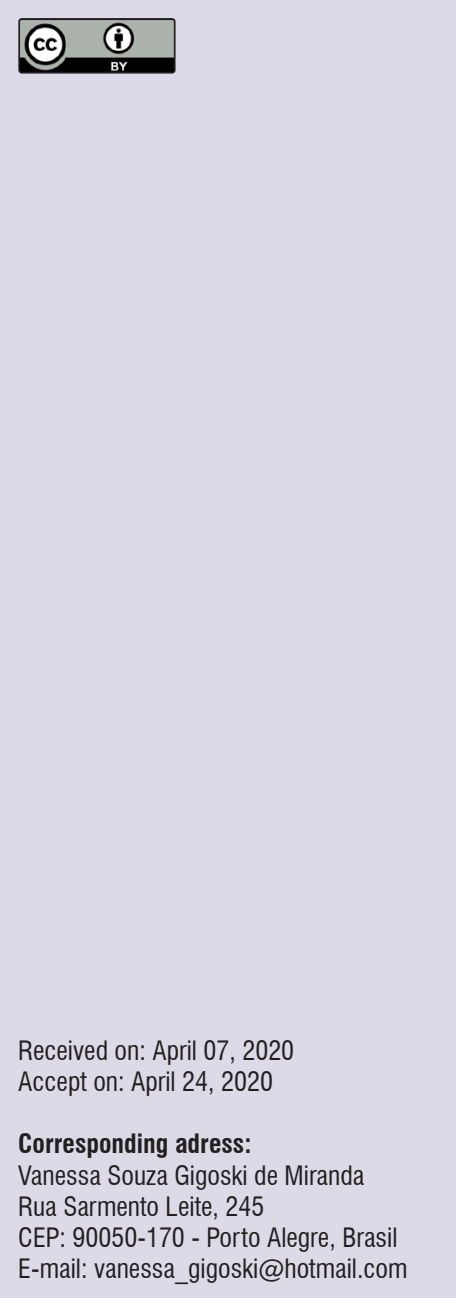

The relevance of evidence-based practice in speech therapy, and in all areas of health, is currently widely recognized, since it can directly impact the quality of health care ${ }^{1}$. In this context, there is a growing production of systematic reviews, and the search for quality in its development aims to improve the scientific production of speech therapy and its influence on the decision of clinical speech therapists and health managers. Systematic reviews may include meta-analyzes, a statistical technique used to combine the results of different individual studies into a single, metanalytical summary measure ${ }^{2}$.

In 2019, 3 systematic reviews were published in the CEFAC Magazine. Vernier et al. (2019) ${ }^{3}$ searched the literature for results of Newborn Hearing Screening in neonates whose mothers had hypertension and / or diabetes mellitus during pregnancy, describing the 5 studies found. Gibrin et al. (2019) ${ }^{4}$ investigated the association of tinnitus with anxiety and depression in the elderly and found 11 studies, carrying out an exploratory approach to the studies individually. Chimelo et al. $(2019)^{5}$, which aimed to describe the audiological characteristics of patients with Mucopolysaccharidosis, found 8 articles described individually. Although all of these reviews ${ }^{3-5}$ present quantitative data, no meta-analysis was performed in any of the studies.

The importance of describing and exploring data in all areas of knowledge is known, but we identified science by clamoring for quantitative data and objective assessments in speech therapy. Meta-analyzes can be carried out both in systematic reviews of intervention studies and in observational studies, and their performance should be encouraged in order to generate quality evidence for speech therapy. Thus, we discuss below the central points of this statistical analysis and its applicability to evidence-based speech therapy.

In the health area, the most common is a meta-analysis to compare the effects of different technologies (drugs, treatments, procedures, instruments, among others). For these studies to be combined, it is necessary to define which results will be combined, which in general are estimates for measures of effect size, such as difference between means, odds ratio, relative risk, absolute risk reduction and the 
number necessary to treat ${ }^{6}$. However, meta-analyzes can also be used to estimate prevalence, incidences, associated factors and risk factors of the comorbidities addressed, allowing the consensus of epidemiological perspectives of speech-language disorders.

It is important to emphasize that the result of a metaanalysis will have applied meaning, only if the studies that compose it are the result of a systematic review. This is because, following the appropriate methodology of a systematic review, studies are selected that meet eligibility criteria defined a priori ${ }^{7}$, which makes them similar in certain characteristics - including methodological design, patient characteristics, interventions / exposures analyzed -, which considered relevant to the research question of the review.

Even with the application of strict eligibility criteria for the selection of studies, the included studies are different and will differ from each other, in one or many characteristics, which may impact on the effect estimates verified in each study, giving statistical heterogeneity to the estimate of effect accumulated by meta-analysis. For example, a study that is being evaluated for the effectiveness of myofunctional treatment, between groups there may be differences in the characteristics of the selected subjects, between studies there may be differences in age, social class, associated comorbidities, and several confounding factors that may interfere with the effectiveness of a treatment from one study to another. When this happens, and the variability between studies is not just random, studies are classified as heterogeneous ${ }^{6}$.

To identify the heterogeneity in the findings, statistical techniques are applied to check whether the differences observed in the results can be explained or not by chance $^{8}$. The most usual ways of verifying the existence of heterogeneity in meta-analyzes are by Cochran's $Q$ test (which verifies whether the heterogeneity is significant) and by the $I^{2}$ inconsistency statistics by Higgins and Thompson (which estimates the magnitude of the heterogeneity, in percentage). An $\mathrm{I}^{2}$ greater than $50 \%$ indicates substantial heterogeneity and, above $75 \%$, considerable heterogeneity ${ }^{7}$.

There are authors who indicate that the greater the heterogeneity, the greater the question about the validity of combining results ${ }^{8}$. Heterogeneity, however, can be a source of information about the effect of an intervention, and its cause should be investigated whenever possible. This can be done through subgroup analysis or meta-regression. By separating studies into subgroups, it is possible, for example, to identify patients who benefit most from interventions or regional differences in prevalence, and to verify whether a summary measure combining subgroups is informative or not. Meta-regression is used to assess the effect of multiple factors on heterogeneity, respecting a limit of a minimum of ten studies to perform this analysis ${ }^{7,8}$. The exclusion of studies can also correct heterogeneity, however, it should be considered only when there is an evident cause for the study to present a different result from the others and not be based only on the observed result ${ }^{7}$.

Still, the authors can incorporate the unexplained heterogeneity in the calculation of the effect measure, using the random effects model for analysis. Basically, there are two models of effect in meta-analysis: fixed effect and random effects. The models are distinguished in an essential factor: what they are measuring as the effect of an intervention / exposure. The fixedeffect model considers that all studies are measuring a single intervention / exposure effect, and that variation between the result measured between studies is due only to chance, that is, it disregards the existence of heterogeneity. On the other hand, the random effect model considers that an intervention / exposure has different but related effects, thus, not only considering the existence of heterogeneity, but also using it to estimate the average effect of the intervention / exposure?.

In sensitivity analyzes, the data included in the metaanalysis is varied to know the impact of this change on the results, that is, to assess the robustness of the results of the meta-analysis. In it, authors can include only studies with specific characteristics, remove heterogeneous studies or with a high risk of bias. Thus, if the result of the meta-analysis and its conclusions are not significantly affected by the changes, it can be said that there is a high confidence in the result presented ${ }^{7}$.

In conclusion, meta-analysis is especially useful for answering research questions in areas where studies have samples of varying size and conflicting results. Many studies, in speech therapy, are identified with small convenience samples, without sample size calculation, often due to the rarity of the patients' clinical condition, as well as the difficulty of sample homogeneity. However, the use of meta-analysis appears as a possibility to combine the results of the studies, increasing the statistical power and the precision of the estimates, by reducing the standard error of the weighted average effect size between the studies $^{9}$, and providing sufficient evidence to support 
decision-making, or even demonstrate the discrepancies in the speech therapy literature and the need for further studies for the question to be answered.

\section{REFERENCES}

1. Miranda VSG, Marcolino MAZ, Rech RS, Barbosa LR, Fischer GB. Evidence-based speech therapy: the role of systematic revisions. CoDAS. 2019;31(2):e20180167.

2. Fletcher RH, Fletcher SW. Epidemiologia clínica: elementos essenciais; tradução Roberta Marchiori Martins. Porto Alegre, Artmed, 2006.

3. Vernier LS, Castelli CTR, Levandowski DC. T Neonatal hearing screening of newborns of mothers with Diabetic Mellitus and/or hypertension in pregnancy: a systematic literature review. Rev. CEFAC. 2019;21(3):e13717.

4. Gibrin PCD, Ciquinato DSA, Gonçalves IC, Marchiori VM, Marchiori LLM. Tinnitus and its relationship with anxiety and depression in the elderly: a systematic review. Rev. CEFAC. 2019;21(4):e7918.

5. Chimelo FT, Silva LAF, Kim CA, Matas CG. Audiological characteristics in mucopolysaccharidosis: a systematic literature review. Rev. CEFAC. 2019;21(5):e16218.

6. Rodrigues CL, Ziegelmann PK. Metanálise: um guia prático. Rev HCPA. 2010;30(4):436-47.

7. Higgins JPT, Thomas J, Chandler J, Cumpston M, Li T, Page MJ et al (eds). Cochrane Handbook for Systematic Reviews of Interventions version 6.0 (updated July 2019). Cochrane, 2019. Available from www.training.cochrane.org/handbook.

8. Pereira MG, Galvão TF. Heterogeneidade e viés de publicação em revisões sistemáticas. Epidemiol. Serv. Saúde. 2014;23(4):775-8.

9. Cohn LD, Becker BJ. How meta-analysis increases statistical power. Psychological methods. 2003;8(3):243-53. 